\title{
A Human Reaching Movement Model for Myoelectric Prosthesis Control
}

\author{
Go Nakamura ${ }^{*}$, Taro Shibanoki ${ }^{\dagger}$, Yuichiro Honda ${ }^{*}$, Akito Masuda $^{\ddagger}$, Futoshi Mizobe ${ }^{\S}$, Takaaki Chin ${ }^{*}$ and Toshio Tsuji ${ }^{* *}$ \\ ${ }^{*}$ Robot Rehabilitation Center in The Hyogo institute of Assistive Technology, 'Ibaraki University, \\ ${ }^{\ddagger}$ Kinki Gishi Corporation, ${ }^{\S}$ Hyogo Rehabilitation Center Hospital, ${ }^{* *}$ Hiroshima University \\ E-mail: g_nakamura@assistech.hwc.or.jp
}

\begin{abstract}
This paper proposes a reaching movement model for the generation of desired trajectories within a myoelectric prosthesis training system. First, an experiment was performed to observe reaching movements with a non-impaired subject and a myoelectric prosthesis user. Reaching movements made by the prosthesis user were then adopted to construct a model based on a logistic function. The proposed model can be used to generate three trajectory types with a bell-shaped speed profile with the adjustment of only a few parameters.
\end{abstract}

Keywords: Myoelectric prostheses, Motion planning, Logistic function

\section{Introduction}

The prescription of myoelectric prostheses to upperlimb amputees supports everyday living and opens up new possibilities for rehabilitation. However, months of training is needed until such prescription can be made. Before being able to use a myoelectric prosthesis, patients must learn the skills necessary to perform tasks with natural posture (e.g., the direction of grasping approach and the manner of forearm usage) in addition to smooth hand opening/closing [1]. However, there is currently no quantitative evaluation method or training system for prosthesis control with natural posture. Against such a background, the authors sought to support myoelectric prosthesis prescription by developing a training system (Fig. 1). The establishment of an evaluation method and such a system requires the construction of a model capable of generating human reaching movement trajectories. A clinically applicable training system with such a model requires the following features: (1) high adaptability for generation of optimized and other trajectories (as myoelectric prosthesis users do not have the reaching movement of non-impaired people due to wrist-related limitations); (2) adjustability of hand velocity (movement time) in consideration of individual differences and variations in trainees' operational ability; (3) easy and intuitive trajectory adjustment for use in clinically applicable training; and (4) real-time trajectory generation for use in training. A number of studies on human reaching movement have been conducted [2] - [4] to clarify the human motor planning mechanism. By way of example, 
the minimum-jerk model [2], the minimum torquechange model [3] and the minimum-variance model [4] have all been proposed and found to be capable of generating human trajectories accurately. However, the trajectories in these studies were determined via optimization calculation; it is difficult to generate trajectories in real time. For control of machinery such as manipulators and vehicles, real-time trajectory generation models have been proposed [5] - [7]. As an example, Tsuji et al. proposed a real-time trajectory generation model based on an artificial potential field and a time base generator (TBG) involving a scalar signal with a controllable finite duration and a bellshaped velocity profile. However, these models are accompanied by problems such as the difficulty of adjusting the many related parameters, which are determined by trial and error.

This paper proposes a human reaching movement model for myoelectric prosthesis control training. The model has high adaptability and can be used to generate three hand trajectory types (straight-line paths, circulararc paths and S-shaped paths) with a bell-shaped speed profile in near-real time with the adjustment of only a few parameters.

\section{Human Reaching Movement for Myoelectric Prostheses}

\subsection{Experimental conditions}

A monitoring experiment was conducted with a nonimpaired subject (25 years old, male) and an experienced myoelectric prosthesis user (49 years old, male; MyoBock hand) toward the development of a reaching movement model for myoelectric prosthesis control. The prosthesis user was a right upper-limb amputee (amputation site: $14 \mathrm{~cm}$ below the elbow), and had used a myoelectric prosthesis for 14 years. His average score for the Box and Block Test [8] was approximately 40 blocks, which exceeds the average of normal-level users. His performance was more efficient than that of most myoelectric prosthesis users.

The subjects were asked to sit on a chair and pick up a cup from one of nine locations on a table, lift it to their mouth and return it to its original location. Each task

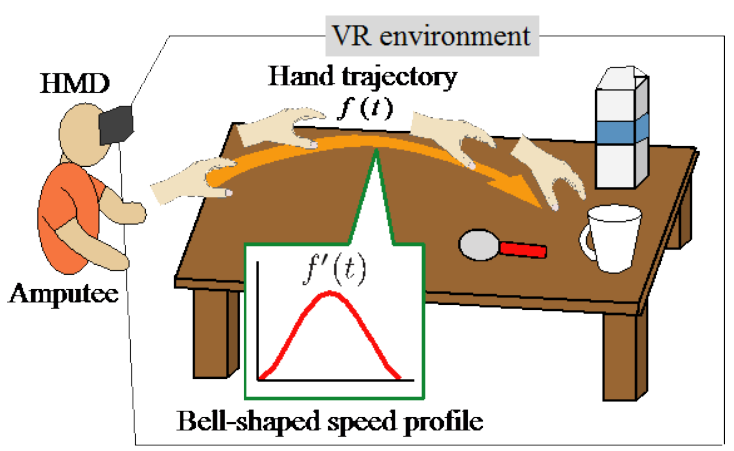

Fig. 1. Concept of the training system for myoelectric prosthesis control using the prosthetic arm trajectory model was repeated five times. A 3D position sensor (Isotrak II, Polhemus Corp.) was attached to the right cubital fossa

of each subject, and their elbow positions $P_{\text {ulwow }}(t)=[x, y, z]^{\mathrm{T}}$ (accuracy: $\pm 2.4 \mathrm{~mm}$ ) and postures (Eulerian angle) $\theta_{\text {elbow }}(t)=[\alpha, \beta, \gamma]^{\mathrm{T}}$ (accuracy: $\pm 0.75^{\circ}$ ) were measured at $60[\mathrm{~Hz}]$. The hand trajectories and hand velocity were evaluated by calculating the hand position $\boldsymbol{P}_{\text {hand }}(t)$ from the measured elbow position and posture. The hand position $P_{\text {hand }}(t)$ is defined as follows:

$P_{\text {hand }}(t)-P_{\text {elbow }}(t)+A\left[\begin{array}{c}\cos \beta \sin \gamma \\ \sin (-\beta) \\ \cos \beta \cos \gamma\end{array}\right]$

Here, $A$ is the forearm length as determined in advance. However, the two subjects had different degrees of freedom in the wrist because the wrist joint of a myoelectric prosthesis is fixed, making evaluation on a level playing field difficult. Accordingly, the location of the wrist joint was defined as the hand position. Hand velocity was estimated using a second order Butterworth low-pass filter (cut-off frequency: 10 [Hz]), and was then calculated using a low-pass differential filter. As the study focused on reaching for and picking up a cup, a two-dimensional trajectory model was constructed as a first step.

\subsection{Results}

Figures 2 shows scenes of reaching tasks for the nonimpaired subject and the myoelectric prosthesis user, respectively. Figure 3 shows trajectories observed in reaching for the cup, with origin coordinates based on the initial hand position. The trajectories represent the average of five trials for each subject. The dashed lines represent the standard deviation for five sessions. The figure shows linear trajectories for both subjects. The 
trajectories of the skilled myoelectric prosthesis user exhibit characteristics similar to those of the nonimpaired subject, but several distinctive differences such as different end points are also observed. The myoelectric prosthesis user also approached the cup placed in the nearest row (lower right, bottom and lower left) after pulling back his elbow, and the trajectories show downward movement. This can be attributed to the fixed wrist joint of the myoelectric prosthesis, which meant that the end points and trajectories for the cup in the nearest row were affected by wrist flexion/extension limitations. The two subjects exhibited different wrist postures at the time of grasping (Fig. 2).

Figure 4 shows hand velocity results based on averages of five trials for each subject. Velocity for the myoelectric prosthesis user was lower than that for the non-impaired subject, but both exhibit bell-shaped velocity profiles. This is because the myoelectric prosthesis user was able to approach the target while opening his hand within the series of motions, and performed the tasks without reducing hand velocity. Figure 2 shows that the myoelectric prosthesis user opened the hand at the start of the approach. These results indicate that the myoelectric prosthesis user performed the tasks with smooth control and hand velocity similar to that of the non-impaired subject.

The hand position and posture data are characterized by a monotonously increasing/decreasing function or a combination thereof, and a similar tendency for all data was observed. The next step in the study was to construct a human reaching movement model using a logistic function.

\section{Trajectory Generation Model for Myoelectric Prostheses}

The proposed model is designed to highlight the appropriate trajectory to a target in a training system. As this study focused on accurately reproducing measured hand trajectory and velocity, the model was not based on the human movement mechanism.

\subsection{Logistic function}

The study involved the proposal of a human reaching movement model using a logistic function.
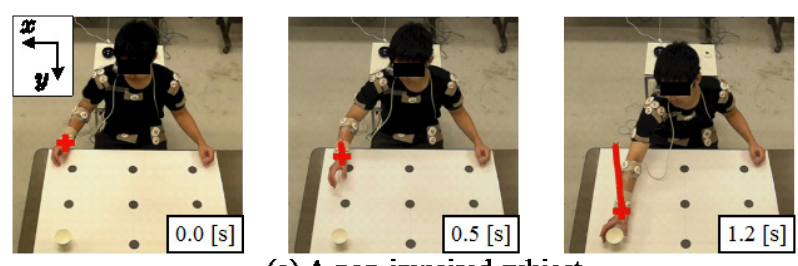

(a) A non-impaired subject
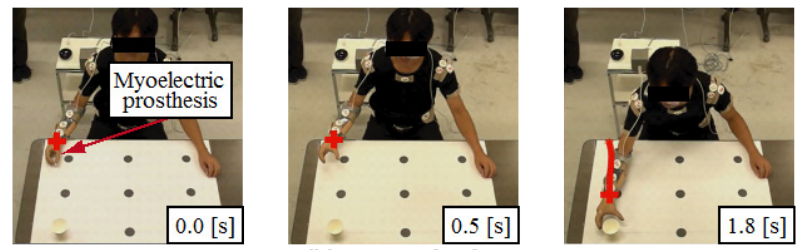

(b) A prosthesis user

Fig. 2. Subjects approaching the cup with upper-right placement

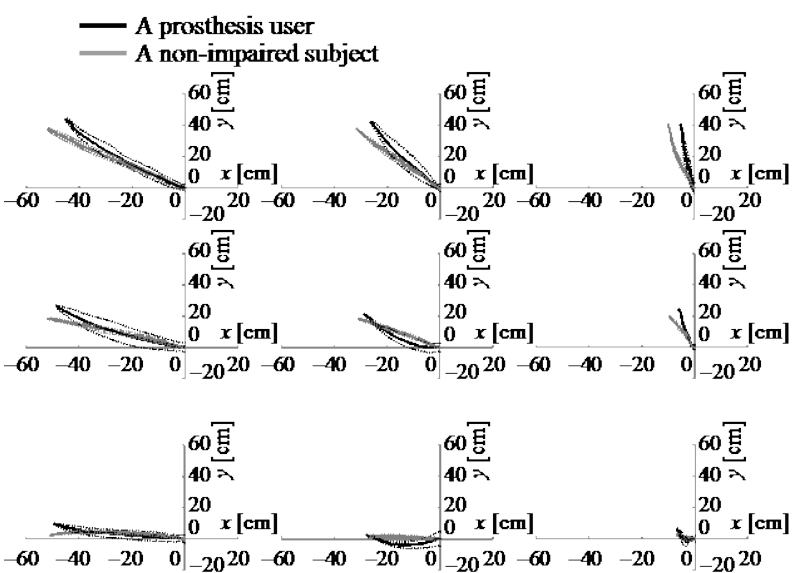

Fig. 3. Measured hand trajectories for the non-impaired subject and myoelectric prosthesis user

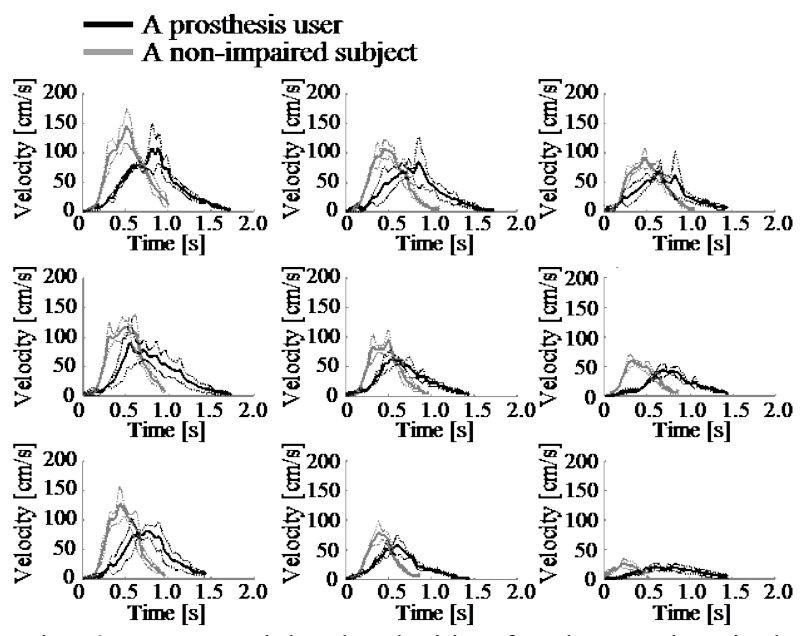

Fig. 4. Measured hand velocities for the non-impaired subject and myoelectric prosthesis user 


$$
\begin{aligned}
& f(t)-\frac{l_{r}}{1+A_{r}\left(c^{-a_{r} t}\right)} \\
& l_{r}-p_{r}^{\text {cnd }}-p_{r}^{\text {start }}
\end{aligned}
$$

Here, $r=(x, y, z, \alpha, \beta, \gamma) \in R^{6}, p_{r}^{\text {slarl }}$ is the start point and $p_{r}^{\text {enc }}$ is the end point. $A_{r}$ is defined as $A_{r}=\epsilon^{a_{r} t_{r}}$. $l_{r}, a_{r}$ and $t_{r}$ are parameters determining function behavior.

\subsection{Spatial trajectory model}

The study involved the proposal of a human reaching movement model using a logistic function.

$x(t)$ and $y(t)$ are defined as

$\left\{\begin{array}{l}x(t)=\frac{l_{x}}{1+A_{x}\left(a_{x} t\right)} \\ y(t)=\frac{l_{y}}{1-A_{y}\left(e^{-a_{y} t}\right)}\end{array}\right.$

To derive an equation for trajectory on the XY plane, the parameter $t$ for (4) and (5) is eliminated. The trajectory on the XY plane can be represented as

$y=\frac{l_{y}}{1+A_{y}\left|\frac{l_{x}-x}{A_{x} x}\right|^{k}}$

Here, $k$ is defined as $k=\frac{a_{y}}{a_{x}}$.

To prevent the denominator from being 0, (6) can be transformed by multiplying the numerator and denominator by $\left|A_{x} x\right|^{k}$ :

$$
y-\frac{l_{y}\left|A_{x} x\right|^{k}}{\left|A_{x} x\right|^{k}\left|A_{y}\right| l_{x}-\left.x\right|^{k}}
$$

Here, the condition is given by $0 \leqq x \leqq l_{x}$.

The proposed model can be used to generate three hand trajectory types (straight-line paths, circular-arc paths and S-shaped paths) featuring a bell-shaped speed profile with the adjustment of only the two ratios of $a_{y} / a_{x}$ and $t_{y} / t_{x}$.

Straight line paths can be generated by setting $l_{x}$ and $l_{y}$ under the conditions of $a_{x}=a_{y}$ and $t_{x}=t_{y}$. Figure 5 shows time histories of spatial trajectory based on the parameters $l_{x}$ and $l_{y}$.

For circular arc paths, trajectories are generated under the conditions of $a_{x}=a_{y}$ and $t_{x} \neq t_{y}$. These paths can be controlled by adjusting only the ratio of $t_{y} / t_{x}$ with $a_{x}-a_{y}$ maintained. Figure 6 shows time histories of spatial trajectory based on the parameters $t_{y} / \ell_{\ldots}$. The curvature gradually increases the farther the ratio of $\iota_{y} / l_{,}$is from 1.0 .

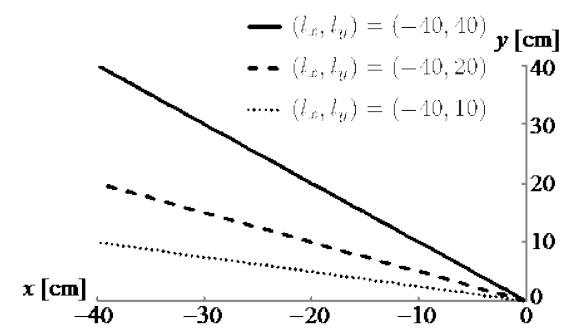

Fig. 5. Straight-line path dependence on the parameters $l$ and $l_{4}$

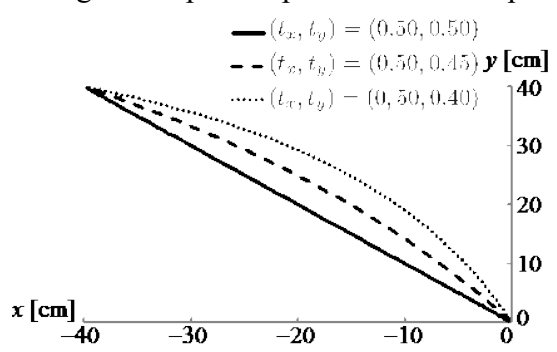

Fig. 6. Circular-arc path dependence on the parameters $t_{y} / t_{x}$

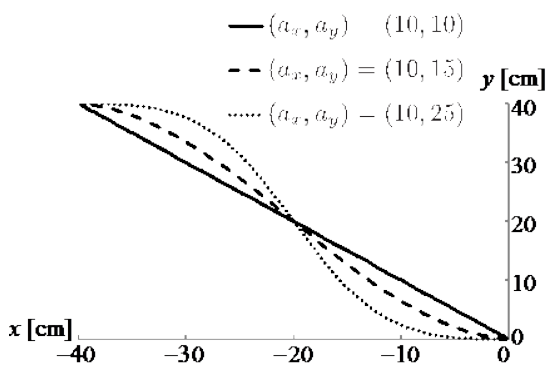

Fig. 7. S-shaped path dependence on the parameters $a_{y} / a_{x}$

For S-shaped paths, trajectories are generated under the conditions of $a_{x} \neq a_{y}$ and $t_{x}=t_{y}$. These paths can be controlled by adjusting only the ratio of $a_{y} / a_{y}$ with $t_{x}=t_{y}$ maintained. Figure 7 shows time histories of spatial trajectory based on the parameters $a_{y y} / a_{t}$. The curvature gradually increases the farther the ratio of $a_{y} / a_{x}$ is from 1.0.

\subsection{Bell-shaped velocity profiles}

The proposed model can be used to generate bellshaped velocity profiles. Single-peaked (unimodal) profiles, double-peaked (bimodal) profiles and other types can be expressed only with adjustment of $a_{y} / a_{*}$ and $l_{y} / l_{x}$.

Unimodal profiles can be generated under the conditions of $t_{x}=t_{y}$. These profiles can be controlled by adjusting only the ratio of $a_{y} / a_{z}$ with $t_{x}=t_{y}$ maintained. Figure 8 shows time histories of spatial trajectory based on the parameters $a_{y} / a_{x}$. Kurtosis gradually increases the farther the ratio of $a_{y} / a_{x}$ is from 1.0. 


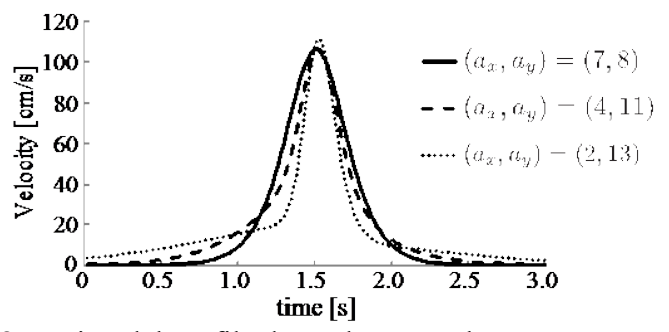

Fig. 8. Unimodal profile dependence on the parameters $a_{y} / a_{x}$

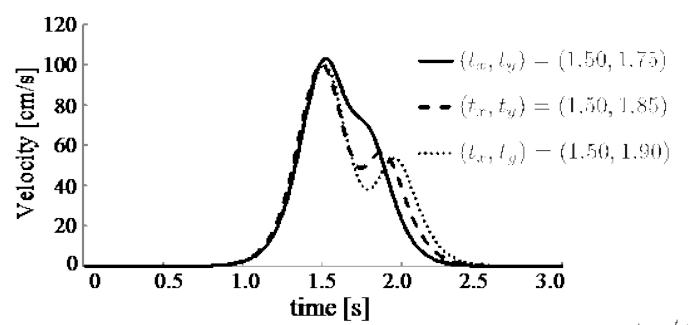

Fig. 9. Bimodal profile dependence on the parameters $t_{y} / t_{x}$

Bimodal profiles can be generated under the conditions of $t_{x} \neq t_{y}$. These profiles can be controlled by adjusting only the ratio of $l_{y} / l_{x}$. Figure 9 shows time histories of spatial trajectory based on the parameters $l_{y} / l_{x}$. The inter-peak distance gradually increases the farther the ratio of $l_{y} / l_{x}$ is from 1.0.

\subsection{Adjustment of movement time}

This subsection outlines how movement time for the proposed model is adjusted. For the logistic function, it is difficult to specify the convergence time because it is not necessarily a finite period. To address this problem, a small arbitrary positive constant $c$ was set. The movement time is defined as $l_{f}$, and the logistic functions $f(t)$ at $t=l_{f}$ can be expressed as

$$
f\left(t_{f}\right)=\frac{l_{r}}{1+A_{r}\left(e^{-a_{r} t_{f}}\right)}=l_{r}(1-\epsilon)
$$

Solving (8) gives the parameters $a_{r}$ and $t_{r}$, which specify the movement time. The issue of their mutual dependence is solved by fixing $t_{r}$ as the time at which the function is $i, 2$. can then be easily estimated from measurement values. In addition, a rational value for $t_{r}$ can be obtained because $t_{r}=t_{f} / 2$ even when it is difficult to obtain a measurement value. Accordingly, $t_{r}$ is fixed in advance and $a_{r}$ is calculated as

$$
a_{r}=\frac{1}{t_{f}-t_{r}} \log \mid \frac{1-\epsilon}{\epsilon}
$$

Here, the condition is given by $t_{r}<t_{f}$ and $0<\epsilon<1$. Specifying $t_{f}$ allows setting of the desired movement time.

\section{Simulation Experiment}

A simulation experiment was conducted to evaluate the proposed model's propensity for hand trajectory generation. $t_{x}$ and $t_{y}$ were set as the times at which the observation values of $x(t)$ and $y(t)$ were nearest to $l_{x} / 2$ and $l_{y} / 2$, respectively. The parameter $t_{f}$ was the measured movement time, and $a_{x}$ and $a_{y}$ were given using (9). $\epsilon$ was set as $\epsilon=0.01$.

Figures 10 and 11 show reaching hand trajectories and hand velocity for each subject, respectively. Solid lines represent hand velocity based on the averages of five trials for observation and generation using the proposed model, and dashed lines represent the standard deviation for five sessions. Although approximation errors are seen with the reaching hand trajectories and hand velocity of the myoelectric prosthesis user for the cup placed in the nearest row, the simulated hand trajectories and hand velocity both correspond to the observations, and the simulated and observed lines are almost identical. Approximation error for the nearest row arises because that the observed time-series data are a combination of an increasing/decreasing function, and the proposed model based on a monotonously increasing/decreasing function was not capable of accurate replication. With this in mind, the authors plan to introduce methods for appropriate combination with the proposed model. From these results, it can be concluded that the proposed model is capable of generating hand trajectories and hand velocity data both for non-impaired subjects and for myoelectric prosthesis users.

\section{Conclusion}

This paper proposes a human reaching model for myoelectric prosthesis control. In the study, the authors performed an experiment to observe reaching movements with a non-impaired subject and a myoelectric prosthesis user. The distinctive characteristics of reaching movements made by the prosthesis user were identified and adopted to support the construction of a model. A simulation experiment was also performed, with results indicating that the proposed model was capable of successfully generating hand trajectories for both subjects.

In future work, the authors plan to introduce methods for combination with the proposed model and expand it model will also be applied for use with a training system. 

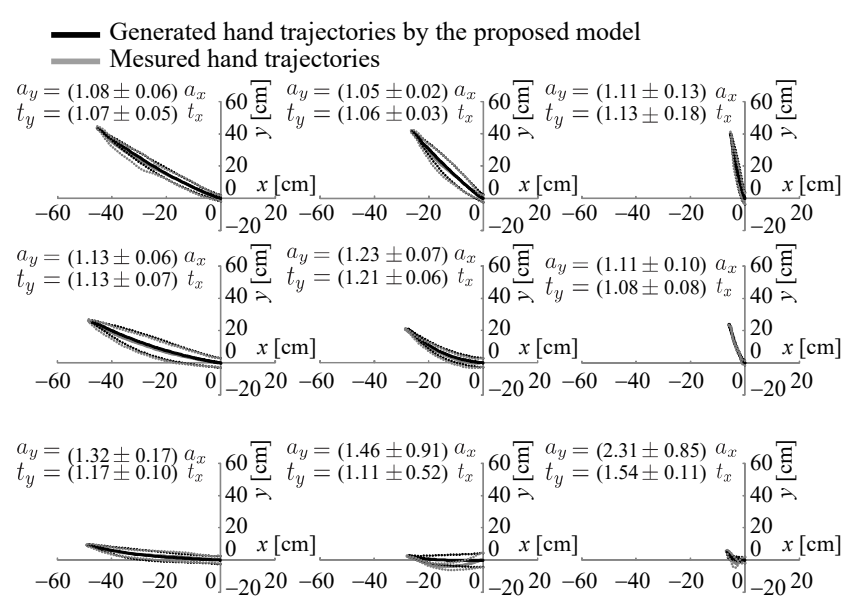

(a): A prosthesis user
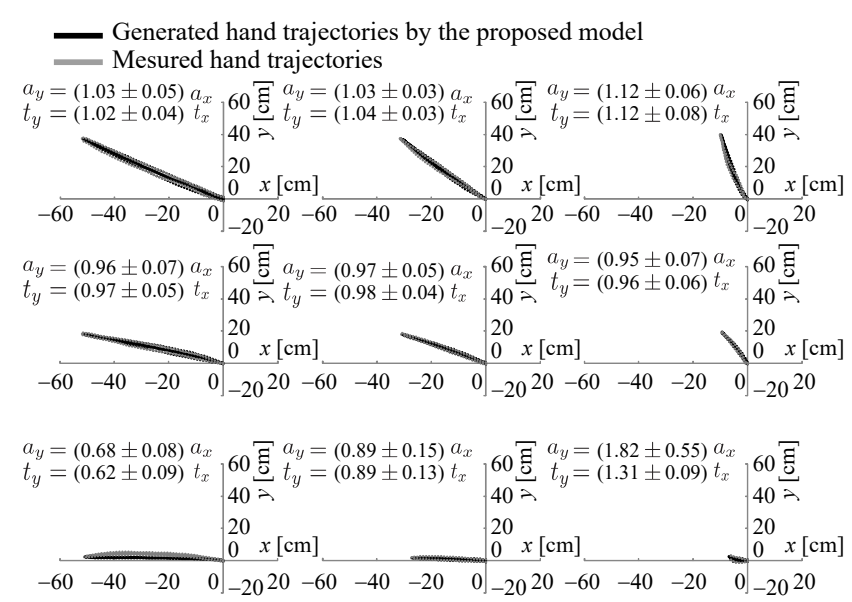

(b): A non-impaired subject

Fig. 10. Observed and simulated hand trajectories for the myoelectric prosthesis user and the non-impaired subject

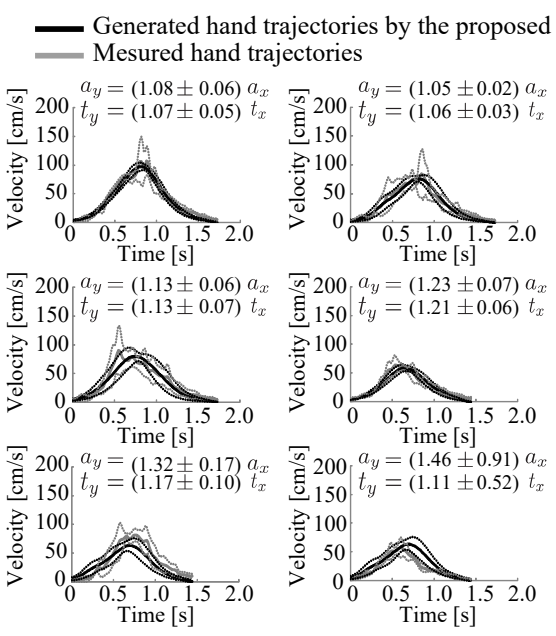

(a): A prosthesis user

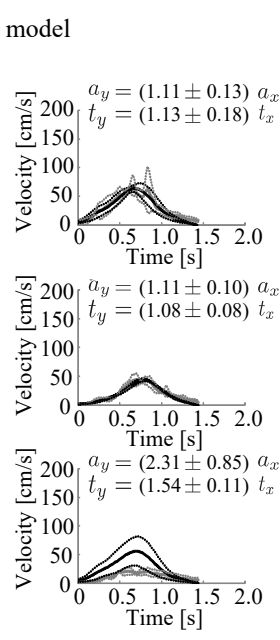

Fig. 11. Observed and simulated hand velocity for the myoelectric prosthesis user and the non-impaired subject

\section{Acknowledgements}

This work was partially supported by a Grant-in-Aid for Young Scientists B Number 26730111.

\section{References}

1. T. Chin, Kinden Gishiyu Kunren Maniyuaru, All-Japan Hospital Publication Association Ltd. (2006), p. 83. (in Japanese).

2. T. Flash and N. Hogan, The Coordination of Arm Movements: An Experimentally Confirmed Mathematical Model, Journal of Neuroscience 5 (7) (1985) 1683-1703.

3. Y. Uno, M. Kawato and R. Suzuki, Formation and Control of Optimal Trajectory in Human Multi-joint Arm Movement, Biol. Cybern. 61 (1989) 89-101.

4. C. M. Harris and D. M. Wolpert, Signal-dependent Noise Determines Motor Planning, Nature 394 (1998) 780-784.

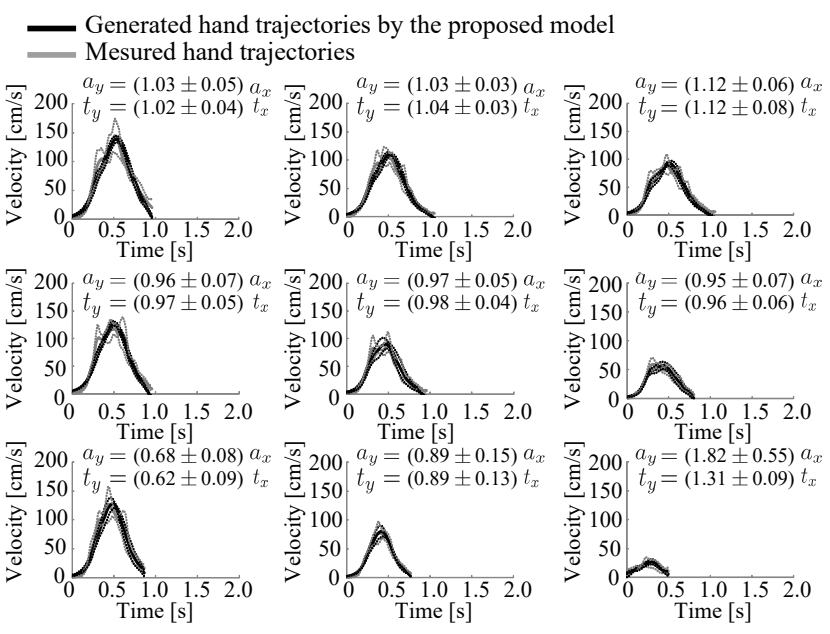

(b): A non-impaired subject

5. A. Badawy, Dual-well Potential Field Function for Articulated Manipulator Trajectory Planning, Alexandria Engineering Journal 55 (2) (2016) 1235-1241.

6. T. Tsuji, Y. Tanaka, P. G. Morasso, V. Sanguineti and M. Kaneko, Bio-mimetic Trajectory Generation of Robots via Artificial Potential Field with Time Base Generator, IEEE Transaction on Systems, Man and Cybernetics 32 (4) (2002) 426-439.

7. T. Weerakoon, K. Ishii and A. A. F. Nassiraei, An Artificial Potential Field Based Mobile Robot Navigation Method to Prevent from Deadlock, Journal of Artificial Intelligence and Soft Computing Research 5 (3) (2015) 189-203.

8. V. Mathiowetz, G. Volland, N. Kashman and K. Weber, Adult Norms for the Box and Block Test of Manual Dexterity, The American Journal Occupational Therapy. 39 (6) (1985) 386-391. 